\title{
环己醇制备环己酮的实验改进
}

陈文涁 ${ }^{*}$, 陈月阳，陈波

南开大学化学学院, 天津 300071

摘要: 对本科生有机化学实验 “环已醇氧化制备环己酮” 进行了改进, 尝试了两种绿色氧化剂: 单过硫酸氢钾(Oxone) 和三氯异氰尿酸(TCCA)。以 Oxone 氧化环己醇制备环己酮的反应时, 发现了一个副产物, 通过气质联用方法确定 了副产物的结构, 并研究了不同条件下的主产物和副产物含量。而利用 TCCA 氧化环己醇制备环己酮时, 可得到高 纯度的目标产物, 其含量达 $93 \%$ 左右, 副产物含量较低, 可以用来替代原有的重铬酸钠氧化环己醇制备环己酮的本 科实验教学。通过引入绿色化学的概念, 加深学生对绿色化学的认识, 感受绿色化学在有机合成中的重要性, 并激 发了他们对科学研究的兴趣。更为重要的是引导学生在化学实验中注意新的发现, 并对新的发现寻根索源, 了解其 原因和过程, 并找到解决的方法。

关键词: 绿色化学; 环已醇; 环已酮; 单过硫酸氢钾; 三氯异氰腿酸

中图分类号: G64; O6

\section{Improvement on Preparation of Cyclohexanone from Cyclohexanol}

\author{
Wenbin Chen *, Yueyang Chen, Bo Chen \\ College of Chemistry, Nankai University, Tianjin 300071, China.
}

\begin{abstract}
In this paper, the organic chemistry experiment "cyclohexanol oxidation to cyclohexanone" was improved by two green oxidants: potassium bisulfate (Oxone) and trichloroisocyanuric acid (TCCA). A by-product was found in the oxidation with Oxone, and the structure of the by-product was determined by GC-MS. The contents of the target product and by-product under different conditions were studied. When TCCA was used as oxidant to prepare cyclohexanone from cyclohexanol, the higher pure target product was obtained (93\%), which can be used to replace the original sodium dichromate oxidation of cyclohexanol to cyclohexanone. Through the introduction of the concept of green chemistry, the students can learn the importance of green chemistry in organic synthesis, and be stimulated the interest in scientific research. It is more important to guide students to pay attention to new discoveries in chemical experiments and to search for the causes or processes of new discoveries, and find out new solutions.
\end{abstract}

Key Words: Green chemistry; Cyclohexanol; Cyclohexanone; Oxone; TCCA

绿色化学又称环境无害化学、环境友好化学、清洁化学, 涉及有机合成、催化、生物化学、分 析化学等学科。氧化反应是有机化学中最重要的基本反应之一, 也是学生需要重点掌握的基本内容 之一, 在氧化反应中引入绿色化学概念, 适应时代主题, 符合社会背景, 充分体现素质教育的目标, 有利于提高学生的综合素质, 增强学生的环保理念 ${ }^{[1-4]}$ 。

仲醇氧化为酮的实验是目前有机化学实验教材中常规的实验内容 ${ }^{[5]}$, 重铬酸盐、氯铬酸吡啶盐

收稿: 2020-11-20; 录用: 2021-01-04; 网络发表：2021-01-18

“通讯作者, Email: wbchen@nankai.edu.cn

基金资助: 南开大学实验教学改革项目(2020) 
(PCC)和重铬酸吡啶盐(PDC)也是常用的氧化试剂, 在实验室中得到了广泛的应用。通过在硅胶、硅 藻土、分子篮或氧化铝上吸附这些试剂来提高产率和便于操作的方法的引入, 增加了它们的受欢迎 程度 ${ }^{[6]}$ 。多年来, 化学教学实验中重视绿色化学要求, 减少危险废物产生, 已成为大家的共识, 为 此, 人们开发了很多新的方法来替代该反应, 包括普遍使用的家用漂白液或商品化次氯酸钠进行氧 化 ${ }^{[7-10]}$, 以及使用过氧化氢水溶液在催化剂条件下的氧化 ${ }^{[6]}$, 这些试剂都比铬酸盐作氧化剂有了很大 程度的改善。尽管漂白液或次氯酸钠是一种安全、方便的氧化试剂, 但用作化学反应时重现性较差, 成功率不高, 而且反应中需要用到醋酸, 沸点较高, 难以去除 ${ }^{[11]}$ 。由于含量不确定, 在进行化学反 应时需要通过滴定标定其含量或者需要加入大大过量的试剂 ${ }^{[12]}$, 同时由于含量低, 反应体系容量较 大。过氧化氢作氧化剂需要加入催化剂, 其制备复杂、成本高, 而且作为一种易爆品, 购买上也受 到一定程度的限制。

国内高校化学学院本科实验教学中, 环己醇氧化制备环己酮是常规的教学实验内容, 多年来一 直使用重铬酸钠和浓硫酸氧化环己醇的方法, 尽管反应稳定, 产率也较高, 但反应过程和反应结果 不符合当前绿色化学和绿色环保的要求, 高校化学教育也要求将绿色化学融入到化学教学中, 既要 将绿色化学内容贯穿到化学理论教育中, 更要将绿色化学方法应用于化学实验教学中。为了利用反 应稳定、安全性高、操作简便的绿色氧化试剂氧化环己醇制备环己酮, 我们对该实验内容进行了改 进, 尝试了单过硫酸氢钾(Oxone)和三氯异氰尿酸(TCCA)进行该反应。Oxone是由过一硫酸氢钾、硫 酸氢钾、硫酸钾结合而成的三合盐, 工业上广泛应用于生活饮用水的消毒和水产养殖的消毒, 而在 有机合成中也是一种常用的氧化剂, 可以氧化伯醇、仲醇得到醛和酮 ${ }^{[12,13]}$, 反应中不散发刺激性蒸 气而造成严重吸入危险, 副产物为不具有毒性而且可回收利用的硫酸钾。TCCA也被广泛应用于饮用 水处理、游泳池消毒、食品防腐和羊毛的非缩水处理等家庭生活和工业生产中, 具有安全性好、易 于操作、耐储存及价格低廉等优点。在有机合成中, 三氯异氧尿酸可作为高效、稳定的氯化试剂和 氧化剂 ${ }^{[14-16]}$, 用作氧化剂可以用来氧化二级醇到酮, 具有反应速度快、操作简便、价格便宜且容易 定量的优点 ${ }^{[17,18]}$ 。

本文利用环境友好的单过硫酸氢钾和三氯异氧尿酸为氧化剂, 氧化环己醇制备环己酮, 代替具 有严重环境污染性和操作危险性高的重铬酸钠/浓硫酸氧化剂, 采用绿色环保的合成方法, 在训练学 生基本操作技能的同时, 又能向学生传授绿色化学和绿色环保的教学思想。

\section{1 实验目的}

通过利用两种不同的氧化剂氧化环己醇制备环己酮, 了解绿色化学的概念, 明确绿色化学的要 求, 掌握和综合运用有机化学实验中各种基本单元操作, 熟悉气相色谱仪进行定性分析的基本操作, 初步掌握反应副产物的分析方法。

\section{2 实验原理}

以环己醇为原料, 以水产养殖、饮用水处理、游泳池消毒等常用的消毒剂为原料, 在低毒溶剂 乙酸乙酯中氧化环己醇制备环己酮, 合成路线如图1所示。

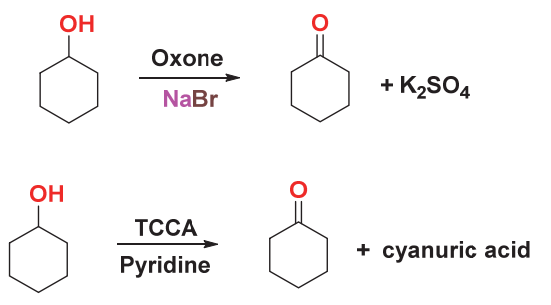

$$
\text { Oxone: } 2 \mathrm{KHSO}_{5} \mathrm{KHSO}_{4} \mathrm{~K}_{2} \mathrm{SO}_{4}
$$

图1 环己酮的合成路线 
从图1可以看出, 当利用Oxone作为氧化剂时, 除了目标产物外, 主要的副产物是硫酸钾, 可以 通过过滤的方法进行回收利用。当利用TCCA作氧化剂时, 主要副产物是氰尿酸和吡啶盐酸盐, 不溶 于反应混合物中, 过滤该白色沉淀后用水洗涤吡啶盐酸盐, 可回收氰尿酸, 采用氯气氯化, 重新生 成三氯异氭尿酸(图2)。由此可见, Oxone和TCCA均不产生对环境污染的废气物, 生成的副产物可以 回收重复利用, 是绿色环保的氧化剂。

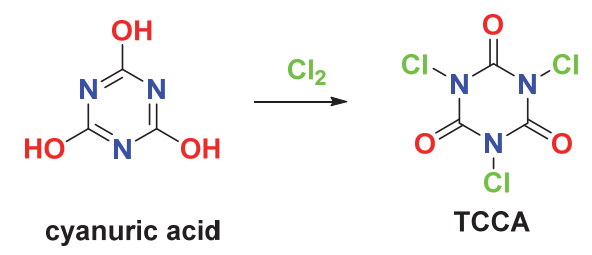

图2 氧尿酸的再生合成路线

\section{3 实验部分}

\section{1 试剂与仪器}

环己醇(天津渤化化学试剂有限公司, 分析纯), 单过硫酸氢钾复合盐(连云港永荣生物科技有限公 司, 分析纯), 三氯异氧脲酸(上海泰坦科技股份有限公司, 90\%), 乙酸乙酯、澳化钠、吡啶、亚硫酸 氢钠、盐酸、硫酸钠、碳酸氢钠(天津市化学试剂六厂, 分析纯)。

84-B型磁力加热搅拌器(山东鄄城华鲁电热仪器有限公司), SHB-III循环水式真空洜(郑州长城科 工贸有限公司), JJ200型电子天平(常熟市双杰测试仪器厂), R-3型旋转蒸发仪(德国布奇), GC-2014 气相色谱仪(日本岛津), HP-5色谱柱 $(30 \mathrm{~m} \times 0.32 \mathrm{~mm}, 0.25 \mathrm{micron})$ 。分析条件: 柱温 $80^{\circ} \mathrm{C}$, 保留 $4 \mathrm{~min}$, 以 $15^{\circ} \mathrm{C} \cdot \mathrm{min}^{-1}$ 速度升至 $250^{\circ} \mathrm{C}$ 保留 $20 \mathrm{~min}$ 。

\section{2 实验步骤}

\subsubsection{Oxone氧化环己醇制备环己酮操作步骤}

$50 \mathrm{~mL}$ 圆底烧瓶中, 加入环己醇 $(1.00 \mathrm{~g}, 10.0 \mathrm{mmol})$, 加入 $10 \mathrm{~mL}$ 酸乙酯, 搅拌溶解后, 向上 述混合物中再加入适量的单过硫酸氢钾复合盐、适量澳化钠、 $3 \mathrm{~mL}$ 水, 将上述混合物在室温下搅拌 $1 \mathrm{~h}$, 反应完毕, 向反应液中加入适量水, 搅拌 $10 \mathrm{~min}$, 待白色固体基本溶解后, 向反应液中加入适 量亚硫酸氢钠固体, 至反应液无色, 并用淀粉-碘化钾试纸测试不显色。将上述反应液转入分液漏斗 中进行分液, 水相用 $10 \mathrm{~mL}$ 乙酸乙酯萃取一次, 合并有机相, 水洗一次, 无水硫酸钠干燥后, 过滤出 干燥剂, 滤液浓缩溶剂得无色透明产品, 并进行气相色谱分析。

\subsubsection{TCCA氧化环己醇制备环己酮操作步骤}

$50 \mathrm{~mL}$ 圆底烧瓶中, 加入环己醇 $(1.00 \mathrm{~g}, 10.0 \mathrm{mmol}), 10 \mathrm{~mL}$ 酸乙酯, 搅拌溶解后, 向上述混 合物中, 加入三氯异氧脲酸 $(4.3 \mathrm{mmol})$ 、吡啶 $(12.0 \mathrm{mmol})$ 室温下搅拌 $1 \mathrm{~h}$, 反应过程中有白色固体生 成, 反应完毕, 进行抽滤, 过滤出生成的氰豚酸和吡啶盐酸盐。滤液, 依次用 $10 \mathrm{~mL} 1 \mathrm{~mol} \cdot \mathrm{L}^{-1}$ 的盐酸 溶液、 $10 \mathrm{~mL}$ 质量分数为 $10 \%$ 的碳酸氢钠溶液、 $5 \mathrm{~mL}$ 饱和氯化钠溶液洗涤, 无水硫酸钠干燥, 过滤出 干燥剂, 滤液浓缩溶剂得无色透明产品, 并进行气相色谱分析。

\section{4 实验结果与讨论}

\section{1 反应条件优化}

一分子Oxone含有两分子的过硫酸氢钾, 因此理论上可以氧化两分子的醇。Oxone氧化醇到醛或 酮的反应, 需要卤代金属盐或铵盐进行催化。我们首先选用了 $\mathrm{NaBr}$ 进行实验, 当环己醇、Oxone的 物质的量之比为 $1: 0.5$ 时, 随着 $\mathrm{NaBr}$ 用量增加, 环己酮的含量并没有增加, 反而有所减少, 但副产物 的含量逐渐增加, 当增加Oxone的用量时, 环己酮的含量基本不变, 原料环己醇转化为副产物的含量 
增加。考虑到Oxone在反应液中为强酸性, 将反应液中和到中性时, 反应混合物中依然有较多的副产 物生成。当用 $\mathrm{NaCl}$ 代替 $\mathrm{NaBr}$ 时, 反应效果更差, 基本得不到环己酮产物, 结果如表1所示, 因此, 上 述结果表明Oxone不适合用来进行氧化环己醇制备环己酮。

表1 Oxone氧化环己醇反应条件篮选结果

\begin{tabular}{|c|c|c|c|c|}
\hline Entry & Cyclohexanol: Oxone : $\mathrm{NaBr}$ 物质的量之比 & 环己醇含量/\% & 副产物(溴代环己酮)含量/\% & 环己酮含量 $/ \%$ \\
\hline 1 & $1: 0.5: 0.3$ & 22.8 & 13.2 & 64.1 \\
\hline 2 & $1: 0.5: 0.4$ & 23.2 & 20.4 & 53.7 \\
\hline 3 & $1: 0.5: 0.5$ & 17.8 & 24.1 & 55.9 \\
\hline 4 & $1: 0.6: 0.3$ & 12.3 & 26.1 & 61.6 \\
\hline 5 & $1: 0.6: 0.4$ & 9.6 & 30.8 & 59.6 \\
\hline 6 & $1: 0.6: 0.5$ & 10.5 & 52.5 & 37.0 \\
\hline 7 & $1: 0.7: 0.05$ & 72.7 & 15.0 & 12.2 \\
\hline 8 & $1: 0.7: 0.05(\mathrm{pH}=7)$ & 57.8 & 24.4 & 17.8 \\
\hline 9 & $1: 0.7: 0.1$ & 65.9 & 16.6 & 17.5 \\
\hline 10 & $1: 0.7: 0.1(\mathrm{pH}=7)$ & 24.7 & 19.3 & 56.0 \\
\hline 11 & $1: 0.7: 0.3$ & 0.0 & 36.2 & 61.5 \\
\hline 12 & $1: 0.7: 0.3(\mathrm{pH}=7)$ & 9.1 & 11.9 & 79.0 \\
\hline 13 & $1: 0.7: 0.4$ & 0.7 & 54.2 & 45.1 \\
\hline 14 & $1: 0.7: 0.5$ & 1.1 & 52.0 & 46.9 \\
\hline
\end{tabular}

当采用 TCCA氧化反应时, 我们在已有的工作基础上, 使用吡啶作该反应的碱, 当环己醇、吡啶 和TCCA的物质的量之比为 $1: 1.2: 0.43$ 时, 原料基本反应完全, 产物的含量达到 $93 \%$, 反应的副产物 含量在 $5 \%$ 左右。当使用碳酸钾作碱时, 反应结果不理想, 生成的产物主要是副产物, 这是因为碳酸 钾的碱性相对较强, TCCA在该反应中同时起到氧化试剂和氯化试剂的作用, 结果如表2所示。

表2 TCCA氧化环己醇反应结果

\begin{tabular}{cccccc}
\hline Entry & 环己醇 $/ \mathrm{TCCA} /$ 碱的物质的量之比 & 碱 & 环己醇含量 $/ \%$ & 副产物含量 $/ \%$ & 环己酮含量 $/ \%$ \\
\hline 1 & $1: 0.43: 1.2$ & 吡啶 & 0 & 5.5 & 93.5 \\
2 & $1: 0.43: 1.2$ & $\mathrm{~K}_{2} \mathrm{CO}_{3}$ & 9.9 & 84.4 & 7.6 \\
\hline
\end{tabular}

\section{2 副产物的结构分析}

当用Oxone作氧化剂氧化环己醇时, 通过气相色谱分析, 产物除了目标产物环己酮之外, 还有另 外一个主要副产物生成, 不同条件下, 副产物的含量有所不同, 通过将反应后的产物进行气相色谱高分辨质谱联用分析, 发现目标产物的分子离子峰98.0726 $\left(\mathrm{M}^{+}\right)$, 符合分子组成 $\mathrm{C}_{6} \mathrm{H}_{10} \mathrm{O}\left(\mathrm{M}^{+} 98.0732\right)$, 副产物的分子离子峰175.9832 $\left(\mathrm{M}^{+}\right), 177.9811\left(\mathrm{M}^{+}+2\right)$, 符合分子组成 $\mathrm{C}_{6} \mathrm{H}_{9} \mathrm{BrO}\left(175.9837\left(\mathrm{M}^{+}\right), 177.9816\right.$ $\left.\left(\mathrm{M}^{+}+2\right)\right)$, 且同位素峰 $M$ 和 $M+2$ 比例约为 $1: 1$, 因此确定副产物的结构是溴代环己酮, GC-MS结果 如图3所示。

由于Oxone中的过一硫酸氢钾有较强的氧化性, 氧化溴离子生成次溴酸, 次澳酸氧化环己醇的 差基得到目标产物环己酮; 同时过一硫酸氢钾可氧化 $\mathrm{Br}^{-}$生成 $\mathrm{Br}_{2}$, 而 $\mathrm{Br}_{2}$ 进一步对酮的 $\alpha$-位进行溴代, 因此生成了溴代环己酮, 如图4所示。由于TCCA氧化环己醇是在碱性环境中, 抑制了环己酮的进一 步氯代，因此反应产物中氯代环己酮的含量较低。 


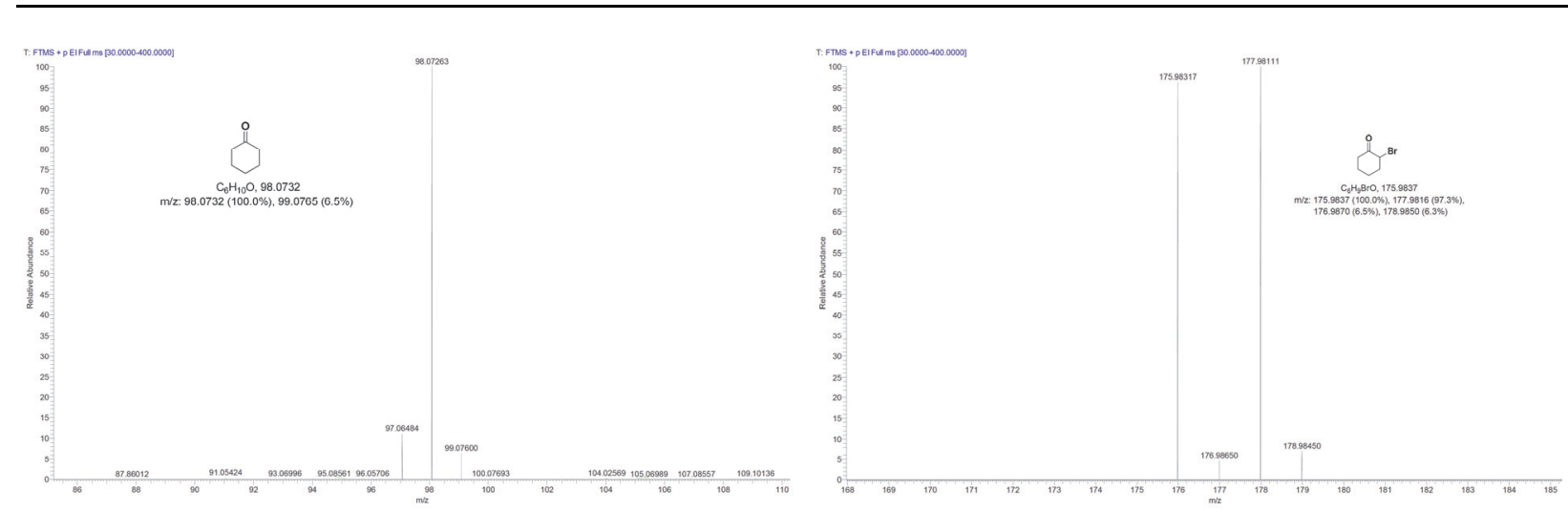

图3 Oxone氧化环己醇反应产物气相色谱-高分辨质谱联用结果

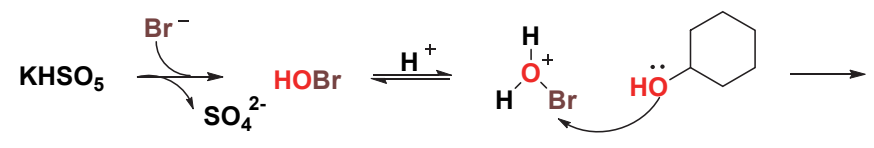

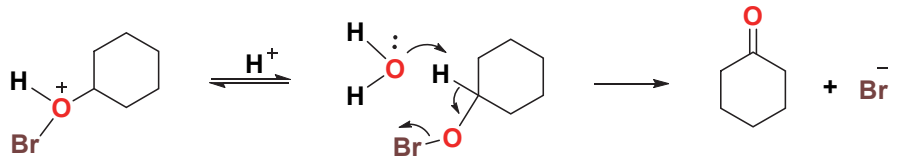<smiles>O=C1CCCCC1CCCCC(Br)(Br)C(=O)O</smiles>

图4 环已酮和澳代环已酮的生成机理

\section{3 产物环己酮的气相含量结果}

不同氧化剂氧化环己醇的 GC结果如图 5所示, 当用Oxone/ $\mathrm{NaBr}$ 作氧化剂时, 产物为环己酮和 溴代环己酮的混合物 (图 5C), 当用 TCCA作氧化剂时, 主要产物为环己酮, 副产物含量约为 $5 \%$ 左右 (图5D), 因此TCCA是一种合适的氧化环己醇制备环己酮的氧化剂。

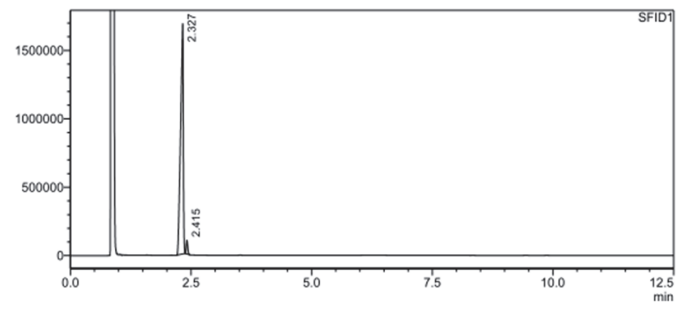

(A) 环己醇原料

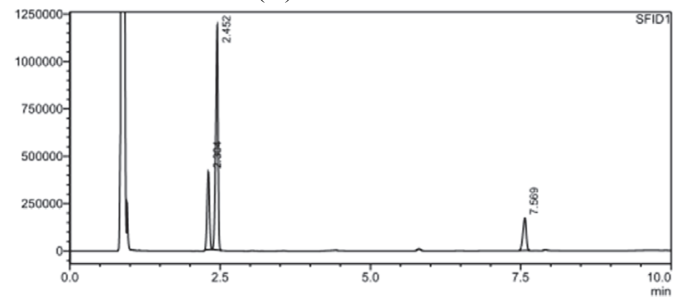

(C) Oxone氧化环己醇反应结果

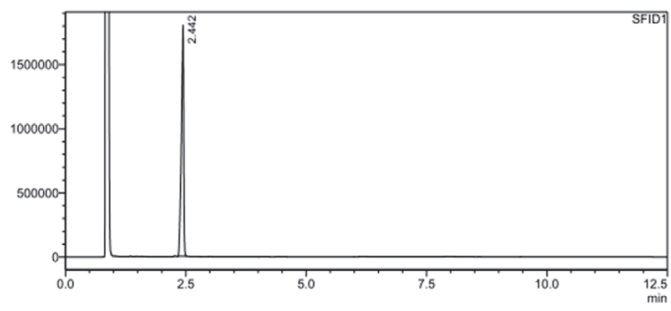

(B) 环己酮标样

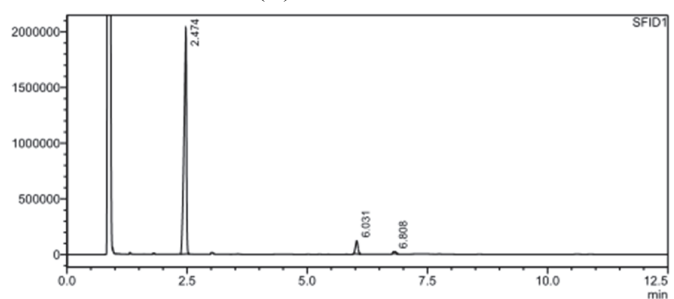

(D) TCCA氧化环己醇反应结果

图5 Oxone和TCCA氧化环己醇反应气相色谱结果图 


\subsection{TCCA氧化环己醇和重铬酸钾氧化环己醇的反应外观及后处理比较}

利用TCCA氧化环己醇制备环己酮时, 反应混合物为白色, 后处理只需要进行过滤和浓缩溶剂即 可, 而重铬酸钠氧化环己醇制备环己酮时, 反应混合物呈深墨绿色, 反应后处理时, 需要利用水蒸 气蒸馏后分液, 并再次进行蒸馏, 方可得到产物, 反应液外观比较如图6所示。因此无论从反应条件、 反应液外观、后处理过程和反应结果均可以认为TCCA是一种绿色氧化环己醇制备环己酮的方法。

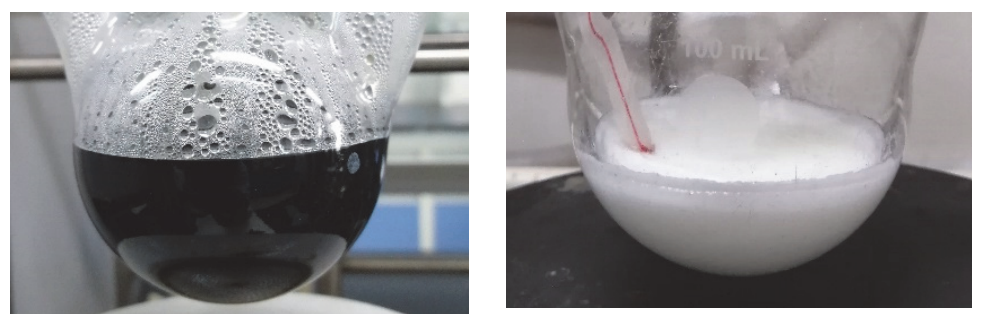

图6 重铬酸钠氧化环己醇(左)和TCCA氧化环己醇(右)反应混合物外观比较 电子版为彩图

\section{5 结语}

通过实验研究了单过硫酸氢钾(Oxone)和三氯异氰尿酸(TCCA)为氧化剂氧化环己醇制备环己 酮, 结果表明Oxone氧化环己醇时, 有副产物溴代环己酮生成, 并进一步通过气相色谱-高分辨质谱 联用仪验证了副产物的结构; 而TCCA为氧化剂氧化时, 可以得到高纯度的环己酮, 是一种优异的氧 化环己醇的绿色氧化剂, 具有安全、稳定、易得、价格便宜的特点。该实验具有反应速度快、操作 简单、收率高、重现性好、反应后的副产物能够回收利用等优点, 能够代替传统的重铬酸钠和硫酸 作为氧化剂氧化环己醇的本科生有机化学实验。通过比较两种不同氧化剂的反应结果, 能够传授绿 色化学的概念, 培养学生认真观察反应过程中出现的异常情况, 并学会通过实验方法解释和验证实 验结果。

\section{参 考 文 献}

[1] 石先䒯. 大学化学, 2015, 30 (5), 26.

[2] 杜欣, 王天泽, 陈渡文, 于景成, 沙广燕. 大学化学, 2020, 35 (4), 19.

[3] 张颖, 许海, 周屹博, 贾琼, 权新军. 化学教育(中英文), 2019, 40 (8), 13.

[4] 徐蓓华, 周子牛, 周惠燕. 化学教育(中英文), 2016, 37 (16), 52.

[5] 邱晓航, 李一峻, 韩杰, 尚贞锋. 基础化学实验. 北京: 科学出版社, 2017: 245.

[6] Hulce, M.; Marks, D. W. J. Chem. Educ. 2001, 78 (1), 66.

[7] Stevens, R. V.; Chapman, K. T.; Weller, H. N. J. Org. Chem. 1980, 45 (10), 2030.

[8] Zuczek, N. M.; Furth, P. S. J. Chem. Educ. 1981, 58 (10), 824.

[9] Mohrig, J. R.; Nienhuis, D. M.; Linck, C. F.; Van Zoeren, C.; Fox, B. G.; Mahaffy, P. G. J. Chem. Educ. 1985, 62 (6), 519.

[10] Straub, T. S. J. Chem. Educ. 1991, 68 (12), 1048.

[11] Vitaku, E.; Christie, H. S. J. Chem. Educ. 2019, 96 (5), 1042.

[12] Lang, P. T.; Harned, A. M.; Wissinger, J. E. J. Chem. Educ. 2011, 88 (5), 652.

[13] Koo, B. S.; Lee, C. K.; Lee, K. J. Synth. Commun. 2002, 32 (14), 2115.

[14] 宗吴中, 胡雨来, 王小平, 刘佳欣, 黄丹风, 王克虎. 有机化学, 2019, 39 (5), 1396.

[15] Gaspa, S.; Carraro, M.; Pisano, L.; Porcheddu, A.; De Luca, L. Eur. J. Org. Chem. 2019, 22, 3544.

[16] Tilstam, U.; Weinmann, H. Org. Process Res. Dev. 2002, 6 (4), 384.

[17] Dip, I.; Gethers, C.; Rice, T.; Straub, T. S. J. Chem. Educ. 2019, 96 (1), 183.

[18] Hiegel, G. A.; Nalbandy, M. Synth. Commun. 1992, 22 (11), 1589. 